\title{
Metabolic and biochemical changes in streptozotocin induced obese-diabetic rats treated with Phyllanthus niruri extract
}

\begin{abstract}
Herbal medicine has been proven to be an effective therapy offering a variety of benefits, such as moderate reduction in hypoglycemia, in the treatment and prevention of obesity and diabetes. Phyllanthus niruri has been used as a treatment for diabetes mellitus. Herein, the induction of type 2 diabetes in Sprague-Dawley rats was achieved by a low dose of streptozotocin (STZ) $(25 \mathrm{mg} / \mathrm{kg} \mathrm{bw})$. Here, we evaluated the in vivo antidiabetic properties of two concentrations ( 250 and $500 \mathrm{mg} / \mathrm{kg} \mathrm{bw}$ ) of P. niruri via metabolomics approach. The administration of $500 \mathrm{mg} / \mathrm{kg}$ bw of P. niruri extract caused the metabolic disorders of obese diabetic rats to be improved towards the normal state. The extract also clearly decreased the serum glucose level and improved the lipid profile in obese diabetic rats. The results of this study may contribute towards better understanding the molecular mechanism of this medicinal plant in managing diabetes mellitus.
\end{abstract}

Keyword: Phyllanthus niruri; ${ }^{1} \mathrm{H}$ NMR-based metabolomics; Diabetes mellitus; Multivariate data analysis; Metabolic pathway 